\title{
The Many-Valued Logic of Quantum Mechanics
}

\author{
Jarosław Pykacz ${ }^{1}$ (1)
}

Received: 30 December 2018 / Accepted: 7 February 2019 / Published online: 10 April 2019

(C) The Author(s) 2019

\begin{abstract}
It is shown that any quantum logic in the Birkhoff - von Neumann sense that possesses an ordering set of probability measures can be isomorphically represented as a particular kind of infinite-valued Łukasiewicz logic with partially defined conjunction and disjunction. It is argued that such non-classical features of $\mathrm{BvN}$ quantum logic as lack of distributivity or validity of the orthomodular law follow from properties of Łukasiewicz conjunction and disjunction.
\end{abstract}

Keywords Quantum logic · Many-valued logic · Łukasiewicz logic

\section{Introduction}

In 1936 Garrett Birkhoff and John von Neumann in their seminal paper 'The logic of quantum mechanics' [2] argued that the structure of a set of dichotomic ('yes-no') propositions about properties of quantum objects, usually called 'quantum logic', which is an orthomodular lattice, differs from the structure of a set of such propositions pertaining to classical objects, in which case it is a Boolean algebra. Nevertheless, since after experimental checking any such dichotomic proposition occurs to be either true or false, quantum logic in the $\mathrm{BvN}$ sense is generally treated as 2-valued logic, non-classical because of lack of distributivity which is unrestrictively guaranteed in Boolean algebras.

Among other non-classical logics studied at that time, in general without references to quantum physics, were various kinds of many-valued logics. Although there were several modest attempts at applying many-valued (mostly 3-valued) logic in description of quantum systems (see bibliography collected in [14]), they never reached mathematical maturity of Birkhoff and von Neumann's proposal. As it was noticed by Max Jammer in his book 'The Philosophy of Quantum Mechanics' [4], this line of investigations ceased in the late 1950s.

The author's way towards expressing BvN quantum logic as a particular kind of Łukasiewicz infinite-valued logic originated from Mạczyński's functional representation theorem and fuzzy sets. In 1973 Maciej Mączyński proved [8] that any orthomodular poset with an ordering set of probability measures $S$ (only such orthomodular posets are

Jarosław Pykacz

pykacz@mat.ug.edu.pl

1 Institute of Mathematics, University of Gdańsk, Wita Swosza 57, 80-952 Gdańsk, Poland 
physically interesting models of quantum logics) can be isomorphically represented as a family of functionals that map $S$ into $[0,1]$ and fulfill certain specific conditions. Since any such functional defines a fuzzy subset of $S$, it was natural to try to express Mączyński's conditions in the language of fuzzy set theory. This nontrivial task, undertaken by the author in 1987 [11], was accomplished in 1994 [12].

Since relations between fuzzy sets and infinite-valued logic are the same as relations between traditional ('crisp') sets and 2-valued classical logic, fuzzy set representation of $\mathrm{BvN}$ quantum logic, i. e. an orthomodular lattice or poset with an ordering set of probability measures (states) $S$, allows to represent it further as a family of infinite-valued propositional functions defined on $S$ with globally defined negation and partially defined conjunction and disjunction (see [13], all the results are collected in [14]). In this way BvN quantum logic can be seen as non-classical logic mainly because of its many-valuedness, with lack of distributivity and other non-classical features being derived from it.

The paper is organized as follows: In Section 2 we give very general arguments why description of future experiments performed on quantum objects requires many-valued logic. In Section 3 we recall the original order-theoretic definition of $\mathrm{BvN}$ quantum logic, and Mączyński's functional representation theorem. Section 4 is devoted to the fuzzy set model of BvN quantum logic while in Section 5 we show that any BvN quantum logic with an ordering set of states can be isomorphically represented as a specific infinite-valued logic endowed with the standard many-valued negation and partially defined Łukasiewicz conjunction and disjunction.

\section{Quantum Mechanics Deals with Futura Contingentia, Therefore it Requires Many-Valued Logic}

The problem of futura contingentia, i.e., statements about future non-certain events has been present in European philosphy since Hellenic times. Aristotle considered the famous statement 'There will be a sea battle tomorrow' in De Interpretatione and it seems (cf. [6]) that he treated such statements as neither true nor false, i.e., according to the modern point of view, as not belonging to the domain of classical two-valued logic.

Since classical mechanics is a deterministic theory, the problem of futura contingentia does not concern it: In deterministic theories all the future is strictly determined, so any statement concerning future events is either true or false already at the moment of stating it, although it may not be known which case occurs. All but stubborn believers in nonprobabilistic hidden variable theories agree that quantum mechanics is indeterministic since in most cases the results of future experiments can be predicted only probabilistically, and this is a 'fundamental' fact not caused by lack of knowledge of some parameters, initial conditions, etc. In other words, quantum probabilities are 'ontic' not 'epistemic', i.e., they do not result from our lack of knowledge.

Jan Łukasiewicz, the founding father of modern theory of many-valued logics, argued in his numerous papers [7] that statements about future non-certain events belong to the domain of many-valued logic. Already in 1913 in his valuable but almost forgotten paper [5] he identified non-classical, i.e., different from 0 or 1 , truth values with probabilities that considered statements will turn out to be true (in classical sense) in the future.

This approach perfectly fits to statements concerning future non-certain events in quantum mechanics: Instead of saying 'Probability that a photon will pass through a semitransparent mirror is 0.5 ' one can, following Łukasiewicz, say 'Truth value of a sentence 
"A photon will pass through a semi-transparent mirror" is 0.5 '. Of course when an experiment is completed, this sentence expressed in the past tense: 'A photon passed through a semi-transparent mirror' occurs to be either true or false, which was the reason for treating $\mathrm{BvN}$ quantum logic as 2-valued logic. Let us note that this 'collapse of many-valued logic onto 2-valued logic' that happens when the experiment is completed is no more strange than passing from probability that is different from 0 or 1 while concidering the future event ' $a$ photon will pass through a semi-transparent mirror' to probability that is either 0 or 1 while concidering the past event 'a photon passed through a semi-transparent mirror'. ${ }^{1}$ They are simply two different events and statements that describe them are two different statements.

When we agree that statements about future non-certain events should be analyzed with the use of many-valued logic, we are still facing a problem which of the plethora of various models of many-valued logics should be used. Fortunately in the case of statements concerning results of future experiments on quantum objects the answer is unique: There is exactly one rather specific model of infinite-valued logic endowed with globally defined negation and partially defined conjunction and disjunction such that any BvN quantum logic in the traditional order-theoretic sense can be isomorphically represented as such logic. The rest of the paper is devoted to showing the details of this construction.

\section{Birkhoff - von Neumann Quantum Logic and its Mączyński's Functional Representation}

Since Birkhoff and von Neumann's paper [2] the 'standard' model of quantum logic is the family $\mathcal{L}(\mathcal{H})$ of closed linear subspaces of a Hilbert space used to describe a studied quantum system or, equivalently, the family of operators of orthogonal projections onto these subspaces. These families from the order-theoretic point of view are orthomodular lattices partially ordered, in the first case by the set-theoretic inclusion, and in the second case by the relation $P_{A} \leq P_{B}$ iff $P_{A} P_{B}=P_{A}$. According to the modern approach to quantum logic theory the term 'quantum logic' usually denotes a little bit more general structure, namely orthomodular partially ordered set, and this position is adopted in the present paper.

By a quantum logic we mean an orthocomplemented $\sigma$-orthocomplete orthomodular poset, i.e. a partially ordered set $L$ that contains the smallest element $O$ and the greatest element $I$, in which the orthocomplementation map $\perp: L \rightarrow L$ satisfying the conditions (a)-(c) exists:

(a) $\left(a^{\perp}\right)^{\perp}=a$.

(b) If $a \leq b$, then $b^{\perp} \leq a^{\perp}$.

(c) The greatest lower bound (meet) $a \wedge a^{\perp}$ and the least upper bound (join) $a \vee a^{\perp}$ with respect to the given partial order exist in $L$ and $a \wedge a^{\perp}=O, a \vee a^{\perp}=I$.

Moreover, the $\sigma$-orthocompleteness condition holds:

(d) If $a_{i} \leq a_{j}^{\perp}$ for $i \neq j$ (such elements are called orthogonal and are usually denoted $\left.a_{i} \perp a_{j}\right)$, then the join $\bigvee_{i} a_{i}$ exists in $L$,

and so does the orthomodular identity:

(e) If $a \leq b$, then $b=a \vee\left(a^{\perp} \wedge b\right)=a \vee\left(a \vee b^{\perp}\right)^{\perp}$.

\footnotetext{
${ }^{1}$ Since the past is fully determined, probabilities of all events that happened in the past, i.e., truth values of all statements that concern past events are either 0 or 1 .
} 
According to Birkhoff and von Neumann (cf. also [1, 10]) elements of a quantum logic represent experimentally verifiable dichotomic ('yes-no') propositions about a studied physical system.

Elements $a, b \in L$ are called compatible iff there exist in $L$ pairwise orthogonal elements $a_{1}, b_{1}, c$ such that $a=a_{1} \vee c$ and $b=b_{1} \vee c$. Let us note that compatibility of elements in a Hilbertian quantum logic $\mathcal{L}(\mathcal{H})$ in its 'projector' version means that projectors are compatible according to this definition if and only if they are commuting (see, e.g., [1]).

Probability measure (also called state) on a quantum logic $L$ is a mapping $s: L \rightarrow[0,1]$ such that

(i) $s(I)=1$,

(ii) $s\left(\bigvee_{i} a_{i}\right)=\sum_{i} s\left(a_{i}\right)$ for any sequence of pairwise orthogonal elements of $L$.

The reason for naming probability measures on quantum logics 'states' follows from the fact that in a Hilbertian quantum logic $\mathcal{L}(\mathcal{H})$ by Gleason Theorem these probability measures are in 1-1 correspondence with density operators on a Hilbert space $\mathcal{H}$ that describes a studied quantum system, which in turn actually represent states of the studied quantum system.

A set of states $S$ on a quantum logic $L$ is called ordering (full, order determining) iff $s(a) \leq s(b)$ for all $s \in S$ implies $a \leq b$. Let us note that only quantum logics with ordering sets of states are physically interesting. This is caused by the fact that the only way in which one can establish experimentally the partial order relation between various propositions is to conduct experiments on a system prepared in various states. Therefore, throughout the rest of the paper we shall consider only quantum logics with ordering sets of states.

Maciej Mączyński in [8] proved a theorem that later on occured to form a substantial part of a 'bridge' by which one can pass from $\mathrm{BvN}$ quantum logic in its order-theoretic form to its fuzzy set or many-valued representation. We quote this theorem in the form that appeared in [9].

Theorem 1 Let $S$ be a non-empty set and let $L$ be a set of mappings from $S$ into $[0,1]$ that has the following three properties:

(i) $\quad 0$ (the null function) belongs to $L$

(ii) $a \in L$ implies $1-a \in L$

(iii) for any (finite or countable) sequence $a_{1}, a_{2}, \ldots \in L$ such that $a_{i}+a_{j} \leq 1$ for $i \neq j$ (such functions were called in [8] pairwise orthogonal), we have $a_{1}+a_{2}+\ldots \in L$.

Then $L$ is a quantum logic with respect to the natural partial order of real functions, with orthocomplementation $a^{\perp}=1-a$. Every point $u \in S$ induces a probability measure $m_{u}$ on $(L, \leq, \perp)$, where $m_{u}(a)=a(u)$ for all $a \in L$, and the family of probability measures $\left\{m_{u}: u \in S\right\}$ is ordering.

Conversely, if $\left(L, \leq,^{\perp}\right)$ is a quantum logic with an ordering set $S$ of probability measures, then each $a \in L$ induces a function $\bar{a}: S \rightarrow[0,1]$ where $\bar{a}(m)=m(a)$ for all $m \in S$. The set of all such functions $\bar{L}=\{\bar{a}: a \in L\}$ has properties $(i)-\left(\right.$ iii) and $\left(\bar{L}, \leq,{ }^{\perp}\right)$ is isomorphic to $\left(L, \leq,{ }^{\perp}\right)$.

\section{Fuzzy Set Representation of BvN Quantum Logic}

It was noticed already in [11] that Mączyński's functions that map a set $S$ into [0,1] actually define membership functions of fuzzy subsets of $S$. Therefore, by Theorem 1 any quantum logic $L$ with an ordering set of probability measures $S$ can be expressed in the language 
of fuzzy set theory and then its condition (i) means that the empty set belongs to $L$, while condition (ii) means that if a set belongs to $L$, then its standard fuzzy complement also belongs to $L$. Also a part of condition (iii) can be expressed with the use of standard fuzzy operations: Two fuzzy sets $A$ and $B$ such that their Eukasiewicz intersection defined as:

$$
(A \sqcap B)(x)=\max [A(x)+B(x)-1,0]
$$

is the empty set, were called weakly disjoint by Giles in [3]. This means that the sum of membership functions of two weakly disjoint sets does not exceed 1, so the beginning of condition (iii) could be expressed as follows: 'For any (finite or countable) sequence of pairwise weakly disjoint sets...'. Unfortunately, the algebraic sum of membership functions that appears in the second part of this condition is not a standard fuzzy set operation and even for three pairwise disjoint sets it may exceed 1. This danger does not exist in the case of Łukasiewicz union (also called bounded or truncated sum):

$$
(A \sqcup B)(x)=\min [A(x)+B(x), 1],
$$

which is an associative operation, and the expression for the Łukasiewicz union of a countable sequence of fuzzy sets is as follows:

$$
\left(\bigsqcup_{i} A_{i}\right)(x)=\min \left[\sum_{i} A_{i}(x), 1\right] \text {. }
$$

However, it occured that simple replacement of algebraic sum of membership functions by their bounded sum in condition (iii) of Mączyński's theorem was not sufficient to make a considered family of fuzzy sets a quantum logic. This aim was achieved in [12] at the expense of introducing the fourth condition stating that the empty set is the only set in the studied family that is wekly disjoint with itself. Let us note that this condition in the realm of traditional ('crisp') sets, where weak disjointness boils down to 'ordinary' disjointness, is always fulfilled.

When this fourth condition is added, Mączyński's theorem is fully expressible in the language of fuzzy set theory and takes the following form [12]:

Theorem 2 Any quantum logic $L$ with an ordering set of probability measures $S$ can be isomorphically represented in the form of a family $\mathcal{L}(S)$ of fuzzy subsets of $S$ satisfying the following conditions:

(a) $\mathcal{L}(S)$ contains the empty set.

(b) $\mathcal{L}(S)$ is closed with respect to the standard fuzzy set complementation.

(c) $\mathcal{L}(S)$ is closed with respect to the countable Eukasiewicz unions of pairwise weakly disjoint sets.

(d) The empty set is the only set in $\mathcal{L}(S)$ that is weakly disjoint with itself.

Conversely, any family of fuzzy subsets of an arbitrary universe $\mathcal{U}$ satisfying conditions (a)-(d) is a quantum logic partially ordered by the inclusion of fuzzy sets, with the standard fuzzy set complementation as orthocomplementation, the orthogonality of the elements coinciding with their weak disjointness, and an ordering set of probability measures generated by points of the universe $\mathcal{U}$ according to the formula

$$
s_{x}(A)=A(x) \text { for all } x \in \mathcal{U} \text {. }
$$

Let us note that it follows from the condition (d) that the only fuzzy subsets in the structure described above that have constant membership functions are the empty set and its 
complement - the universe. Indeed, if there is in the structure any set with constant membership function, then either this set, or its complement is weakly disjoint with itself which, by condition (d), means that its membership function either everywhere equals 0 or everywhere equals 1 .

\subsection{Two Pairs of Binary Operations}

Although by Theorem 2 fuzzy set representation $\mathcal{L}(S)$ of any BvN quantum logic $L$ with an ordering set of probability measures $S$ is characterized by fuzzy set operations, in particular Łukasiewicz intersection (1) and union (2), by the second part of this theorem it is still a partially ordered set, so it is still equipped with order-theoretic operations of meet and join. Relations between these two pairs of operations were studied in [13] and are summarized in the following theorem (see also [14]).

Theorem 3 Let $\mathcal{L}(\mathcal{U})$ be a family of fuzzy subsets of an arbitrary universe $\mathcal{U}$ satisfying conditions (a)-(d) of Theorem 2, and let $A, B \in \mathcal{L}(\mathcal{U})$. Then $A \sqcap B \in \mathcal{L}(\mathcal{U})$ iff $A \sqcup B \in$ $\mathcal{L}(\mathcal{U})$, and in this case $A$ and $B$ are compatible, $A \sqcap B=A \wedge B$, and $A \sqcup B=A \vee B$.

It follows from this theorem that in general Łukasiewicz intersection (1) and union (2) are in $\mathcal{L}(\mathcal{U})$ defined only partially, even if $\mathcal{L}(\mathcal{U})$ is a lattice in which meet and join for any pair of elements exist. Indeed, if Łukasiewicz intersection (1) and union (2) were in any $\mathcal{L}(\mathcal{U})$ defined globally, then all elements would be compatible, so any $\mathcal{L}(\mathcal{U})$ would be a Boolean algebra. This also shows that the existence in $\mathcal{L}(\mathcal{U})$ of meet or join of any elements is not a sufficient condition for the existence of their Łukasiewicz union or intersection, although the opposite is true.

It was noticed in [16] that by transposition of an implication: 'if $A \sqcap B, A \sqcup B \in \mathcal{L}(\mathcal{U})$, then $A$ and $B$ are compatible', one obtains corollary:

Corollary 1 If $A, B \in \mathcal{L}(\mathcal{U})$ are not compatible, then $A \sqcap B, A \sqcup B$ are not defined in $\mathcal{L}(\mathcal{U})$.

This again shows that Łukasiewicz intersection (1) and union (2) are, in general, defined in $\mathcal{L}(\mathcal{U})$ only partially. Let us stress that in view of well-known interpretational difficulties yielded by the original $\mathrm{BvN}$ hypothesis that meets and joins are proper algebraic representations of conjunctions and disjunctions (cf. [1,2]), the fact that Łukasiewicz operations are in quantum logics not globally defined is rather a virtue than a drawback (see discussion in [16]).

It is easy to show, e.g. by taking $A(x)=B(x)=C(x)=0.5$, that Łukasiewicz intersection (1) and union (2) are, in general, not distributive, i.e., that in general

$$
(A \sqcap B) \sqcup C \neq(A \sqcup C) \sqcap(B \sqcup C), \quad(A \sqcup B) \sqcap C \neq(A \sqcap C) \sqcup(B \sqcap C) .
$$

However, the formal counterpart of the orthomodular identity, with meets and joins replaced, respectively, by Łukasiewicz conjuctions and disjunctions, holds for any pair of fuzzy sets such that $A \subseteq B$ :

Lemma 1 If $A \subseteq B$, then $B=A \sqcup\left(A^{\prime} \sqcap B\right)=A \sqcup\left(A \sqcup B^{\prime}\right)^{\prime}$.

Proof For any $x$ we have: $\left[A \sqcup\left(A^{\prime} \sqcap B\right)\right](x)=\min \left[A(x)+\left(A^{\prime} \sqcap B\right)(x), 1\right]=\min [A(x)+$ $\left.\max \left[A^{\prime}(x)+B(x)-1,0\right], 1\right]=\min [A(x)+\max [1-A(x)+B(x)-1,0], 1]=$ 
$\min [A(x)+\max [B(x)-A(x), 0], 1]=\min [A(x)+B(x)-A(x), 1]=B(x)$. The second equality follows from the fact that Łukasiewicz intersection (1) and union (2) together with the standard fuzzy complementation form a de Morgan triple, so the proof is finished.

Let us now assume that $\mathcal{L}(\mathcal{U})$ is a family of fuzzy subsets of an arbitrary universe $\mathcal{U}$ satisfying conditions (b) and (c) of Theorem 2, and let $A, B \in \mathcal{L}(\mathcal{U})$ be such that $A \subseteq B$. This means that for any $x \in \mathcal{U}$ we have $A(x) \leq B(x) \Leftrightarrow A(x)-B(x) \leq 0 \Leftrightarrow A(x)+1-$ $B(x) \leq 1 \Leftrightarrow A(x)+B^{\prime}(x) \leq 1 \Leftrightarrow A \sqcap B^{\prime}=\emptyset$. This by condition (c) of Theorem 2 means that $A \sqcup B^{\prime} \in \mathcal{L}(\mathcal{U})$, which in turn by condition (b) of this theorem and de Morgan identity means that $\left(A \sqcup B^{\prime}\right)^{\prime}=A^{\prime} \sqcap B \in \mathcal{L}(\mathcal{U})$. Now let us note that because of associativity of Łukasiewicz intersection we have $A \sqcap\left(A^{\prime} \sqcap B\right)=\left(A \sqcap A^{\prime}\right) \sqcap B=\emptyset \sqcap B=\emptyset$, so by condition (c) of Theorem $2 A \sqcup\left(A^{\prime} \sqcap B\right) \in \mathcal{L}(\mathcal{U})$. These observations together with Lemma 1 yield the following lemma:

Lemma 2 Let $\mathcal{L}(\mathcal{U})$ be a family of fuzzy subsets of an arbitrary universe $\mathcal{U}$ satisfying conditions $(b)$ and $(c)$ of Theorem 2. Then in $\mathcal{L}(\mathcal{U})$ the formal counterpart of the orthomodular identity holds and all sets that appear in it belong to $\mathcal{L}(\mathcal{U})$.

\section{BvN Quantum Logic as Infinite-Valued Logic}

Relations between fuzzy sets and many-valued logics are exactly the same as relations between traditional ('crisp') sets and classical two-valued logic. For example, set-theoretic complementation is related to logical negation, intersection of sets is related to conjunction, and union of sets is related to disjunction in the same way in both cases:

$$
\begin{gathered}
A^{\prime}=\{x: x \notin A\}, \\
A \cap B=\{x: a \in A \text { and } a \in B\}, \\
A \cup B=\{x: a \in A \text { or } a \in B\} .
\end{gathered}
$$

Of course in the case of fuzzy sets and many-valued logics the specific forms of these expressions depend on the adopted model of complementation/negation, intersection/conjunction, and union/disjunction. Because of Theorem 2, we are bound to choose the original Łukasiewicz negation

$$
\tau(\neg p)=1-\tau(p),
$$

where $\tau(p) \in[0,1]$ is a truth value of a proposition $p$, and according to the idea expressed by Łukasiewicz in his 1913 paper [5] this number is identified with the probability that the proposition $p$ will turn out to be true when suitable experiment is completed. It also follows from Theorem 2 that we should choose as conjunction and disjunction operations that are logical counterparts of fuzzy set operations defined by formulas (1) and (2): ${ }^{2}$

$$
\begin{gathered}
\tau(p \sqcap q)=\max [\tau(p)+\tau(q)-1,0], \\
\tau(p \sqcup q)=\min [\tau(p)+\tau(q), 1] .
\end{gathered}
$$

Before we shall pass to the many-valued model of $\mathrm{BvN}$ quantum logic, let us stress that actually this logic does not deal with propositions but with propositional functions the domain of which is the set of states of a studied physical system. To show this let us consider

\footnotetext{
${ }^{2}$ These logical connectives are nowadays called Eukasiewicz conjunction and disjunction, although they were never studied by Łukasiewicz in his original papers (cf. [7]).
} 
a statement 'A photon will pass through a linear polarizer'. Obviously the truth value of this statement depends on the polarization state of the photon, and various polarization states yield various truth values of this statement. It follows from the remark after Theorem 2 that there are only two constant propositional functions (i.e., actually, propositions) in the studied structure: the always false propositional function $f$ and the always true propositional function $v$, which can be linguistically expressed, for example as: $f=$ 'The studied physical system does not exist' and $v=$ 'The studied physical system exists'.

Let us call two propositional functions $a(\cdot)$ and $b(\cdot)$ exclusive if their Łukasiewicz conjunction (10) is always false, i.e., if for all $x$ in their common domain

$$
\tau((a \sqcap b)(x))=\max [\tau(a(x))+\tau(b(x))-1,0]=0=\tau(f(x)) .
$$

Then we can express Theorem 2 in the language of many-valued logic as follows:

Theorem 4 Any quantum logic $L$ with an ordering set of probability measures $S$ can be isomorphically represented as a family $\Lambda(S)$ of propositional functions defined on $S$ and satisfying the following conditions:

(a) $\Lambda(S)$ contains the always-false propositional function $f$.

(b) $\Lambda(S)$ is closed with respect to Eukasiewicz negation (9).

(c) $\Lambda(S)$ is closed with respect to Eukasiewicz disjunction of pairwise exclusive propositional functions, i.e. if $a(\cdot)_{i} \sqcap a(\cdot)_{j}=f$ for $i \neq j$, then $\sqcup_{i} a(\cdot)_{i} \in \Lambda(S)$.

(d) The always-false propositional function $f$ is the only propositional function in $\Lambda(S)$ that excludes itself, i.e., for any $a(\cdot) \in \Lambda(S)$, if $a(\cdot) \sqcap a(\cdot)=f$, then $a(\cdot)=f$.

Conversely, any family of many-valued propositional functions defined on a common domain D and satisfying conditions $(a)-(d)$ is a quantum logic in Birkhoff - von Neumann sense whenever we identify propositional functions that assume the same truth value for every argument in their common domain D. This family is partially ordered by pointwise partial order of propositional functions:

$$
a(\cdot) \leq b(\cdot) \text { iff } \tau(a(x)) \leq \tau(b(x)) \text { for all } x \in D,
$$

with Łukasiewicz negation (9) as orthocomplementation, orthogonality of elements coinciding with their exclusiveness, and an ordering set of probability measures being generated by arguments in the common domain $D$ according to the formula

$$
s_{x}(a(\cdot))=\tau(a(x)) \text { for all } x \in D .
$$

Let us note that formula (13) actually means that partial order between propositional functions is defined by Łukasiewicz implication defined by him (cf. [7]) as follows:

$$
\tau(p \rightarrow q)=\min [1-\tau(p)+\tau(q), 1] .
$$

Indeed, $\tau(a(x)) \leq \tau(b(x))$ for all $x \in D$ means that $\min [1-\tau(a(\cdot))+\tau(b(\cdot)), 1]=1$, so condition (13) can be replaced by the statement:

$$
a(\cdot) \leq b(\cdot) \text { iff } a(\cdot) \rightarrow b(\cdot)=v
$$

i.e., a propositional function $a(\cdot)$ precedes a propositional function $b(\cdot)$ iff Łukasiewicz implication ' $a(\cdot)$ implies $b(\cdot)$ ' is always true. 


\section{Conjunctions and Disjunctions in the Many-Valued Logic of Quantum Mechanics}

There are no doubts that in Boolean algebras, which are proper models of sets of dichotomic ('yes-no') propositions about properties of classical objects, conjunctions and disjunctions of these propositions are modeled by order-theoretic operations of meet and join. However, even Birkhoff and von Neumann - the founding fathers of quantum logic - in their historic paper [2] doubted whether this idea can be extended to sets of propositions about properties of quantum objects, where some properties are not simultaneously measurable. Discussions on this problem lasted for years (cf. $[1,16])$ but the problem is still open.

Theorem 4 strongly suggests that conjunctions and disjunctions of statements concerning results of future experiments on quantum objects should be modeled by Łukasiewicz conjunction (10) and disjunction (11), not by order-theoretic operations of meet and join. Of course it follows from Corollary 1 that these conjunctions and disjunctions can be formed only when elements of a quantum logic are compatible, i.e., respective properties of quantum objects are simultaneously measurable.

Since it follows from Theorem 3 that if Łukasiewicz operations (10) and (11) are defined they coincide, respectively, with meet and join, this explains why for many years meets and joins were treated as proper order-theoretic models of conjunctions and disjunctions in quantum logics. The results of Section 4.1, when translated from the language of fuzzy sets into the language of many-valued logic, show that such non-classical features of $\mathrm{BvN}$ quantum logic as lack of distributivity or replacement of distributivity laws by a weaker orthomodular law may be seen as following from the many-valuedness of this logic and from properties of Łukasiewicz negation, conjunction, and disjunction.

Let us finish with a remark that the problem of choosing proper mathematical representation of conjunctions and disjunctions is of utmost importance in studying situations of the EPR - Bell - GHZ - etc. types since in all these situations there appear conjunctions of the form: 'The object $A$ has property $x$ AND the object $B$ has property $y$ '. Some investigations in this direction were published in [15].

Open Access This article is distributed under the terms of the Creative Commons Attribution 4.0 International License (http://creativecommons.org/licenses/by/4.0/), which permits unrestricted use, distribution, and reproduction in any medium, provided you give appropriate credit to the original author(s) and the source, provide a link to the Creative Commons license, and indicate if changes were made.

\section{References}

1. Beltrametti, E., Cassinelli, G.: The Logic of Qantum Mechanics. Addison-Wesley, Reading (1981)

2. Birkhoff, G., von Neumann, J.: The logic of quantum mechanics. Ann. Math. 37, 823-843 (1936)

3. Giles, R.: Łukasiewicz logic and fuzzy set theory. Int. J. Man-Machine Stud. 67, 313-327 (1976)

4. Jammer, M.: The Philosophy of Quantum Mechanics. Wiley-Interscience, New York (1974)

5. Łukasiewicz, J.: Die logische Grundlagen der Warscheinlichkeitsrechnung. Kraków (1913). English translation in Ref. [7], pp. 16-63

6. Łukasiewicz, J.: Philosophishe Bemerkungen zu mehrwertigen Systemen des Aussagenkalküls. Comptes rendus des séances de la Société des Sciences et des Lettres de Varsovie. Cl III 23, 51-77 (1930). English translation in Ref. [7], pp. 153-178

7. Łukasiewicz, J.: Selected Works. In: Borkowski, L. (ed.) North-Holland, Amsterdam (1970)

8. Mạczyński, M.J.: The orthogonality postulate in axiomatic quantum mechanics. Int. J. Theor. Phys. 8, 353-360 (1973)

9. Mạczyński, M.J.: Functional properties of quantum logics. Int. J. Theor. Phys. 11, 149-156 (1974) 
10. Pták, P., Pulmannová, S.: Orthomodular Structures as Quantum Logics. Kluwer, Dordrecht (1991)

11. Pykacz, J.: Quantum logics as families of fuzzy subsets of the set of physical states. Preprints of the Second International Fuzzy Systems Association Congress, Tokyo, July 20-25, vol. 2, pp. 437-440 (1987)

12. Pykacz, J.: Fuzzy quantum logics and infinite-valued $Ł$ ukasiewicz logic. Int. J. Theor. Phys. 33, 14031416 (1994)

13. Pykacz, J.: Łukasiewicz operations in fuzzy set and many-valued representations of quantum logics. Found. Phys. 30, 1503-1524 (2000)

14. Pykacz, J.: Quantum Physics, Fuzzy Sets and Logic. Steps Towards Many-Valued Interpretation of Quantum Mechanics. Springer Briefs in Physics. Springer, Cham (2015)

15. Pykacz, J.: Can many-valued logic help to comprehend quantum phenomena. Int. J. Theor. Phys. 54, 4367-4375 (2015)

16. Pykacz, J., Frąckiewicz, P.: The problem of conjunction and disjunction in quantum logics. Int. J. Theor. Phys. 56, 3963-3970 (2017)

Publisher's Note Springer Nature remains neutral with regard to jurisdictional claims in published maps and institutional affiliations. 